\title{
Lower intestinal bleeding secondary to colonic vascular ectasia
}

\author{
SCOTT A. JENKINSON, DO \\ NARCISO S. ALBARRACIN, JR, MD
}

\begin{abstract}
Because multiple lesions, including colonic malignancies, vascular lesions of the colon, and diverticuli may coexist, identifying the cause of lower gastrointestinal bleeding in an elderly patient can be difficult. A case that demonstrates this clinical problem is reported. Right-sided colonic vascular ectasia eventually was detected angiographically and was treated successfully with hemicolectomy. Colonic vascular ectasia is an increasingly recognized cause of gastrointestinal hemorrhage. The pathologic findings, proposed theories of pathogenesis, and clinical association between colonic vascular ectasia and aortic stenosis are discussed.
\end{abstract}

In elderly persons, the incidences of diverticulum, adenocarcinoma, and right-sided vascular ectasia (angiodysplasia) of the colon are increased in comparison to those in younger age groups. These colonic lesions, which are recognized causes of lower intestinal bleeding, may coexist, thus making identification of the one responsible for hemorrhage extremely difficult. The reported case exemplifies this problem.

\section{Report of case}

An 80-year-old woman was admitted with hematochezia and microcytic anemia. Past medical history included hypertension, degenerative joint disease, and appendectomy, many years in the past. Current medications included nalfon, digoxin, and Persantine (dipyridamole). On admission, the hemoglobin and hematocrit values were $4.7 \mathrm{~g} / \mathrm{dL}$ and $16.3 \%$, respectively. A harsh, grade V/VI, systolic ejection murmur heard over the entire precordium was thought to be caused by aortic stenosis. The patient was given six units of packed red blood cells.
Sigmoidoscopy demonstrated an annular, partially obstructing colonic lesion $13 \mathrm{~cm}$ from the anal verge. Aircontrast barium enema showed the same rectosigmoid lesion, as well as several colonic diverticuli on the leftside.

Rectosigmoid colon resection was performed, and a moderately differentiated adenocarcinoma (Astler-Coller [modified Duke's] stage C2) was removed. No further bleeding occurred, and, at discharge, the patient's hemoglobin level was $12 \mathrm{~g} / \mathrm{dL}$.

Two months later, the patient returned to the hospital complaining of weakness. Her hemoglobin and hematocrit values were $6.9 \mathrm{~g} / \mathrm{dL}$ and $22.5 \%$, respectively. A stool guiac test was positive for occult blood. Barium enema demonstrated constriction at the colonic anastomotic site, and recurrence of adenocarcinoma was suspected. However, biopsy failed to demonstrate tumor recurrence.

The serum carcinoembryonic antigen value was 1.05 $\mathrm{ng} / \mathrm{dL}$. The source of the occult blood in the stool was not found. Three units of packed red cells were transfused, and the patient's hemoglobin level at discharge was $11.2 \mathrm{~g} / \mathrm{dL}$.

She was again admitted approximately three months later with lower intestinal bleeding. The hemoglobin level was $5.7 \mathrm{~g} / \mathrm{dL}$, and the hematocrit value was $17.4 \%$. Colonoscopy revealed six to eight small clusters of telangiectatic blood vessels within the ascending and transverse colon, as well as several left colonic diverticuli. Six units of packed red cells were transfused.

Esophagogastroduodenoscopy showed a small superficial duodenal ulcer, which was not bleeding. Two radioisotope ( ${ }^{99 \mathrm{M}} \mathrm{Tc}$ sulfur colloid) blood loss scans failed to localize the colonic hemorrhage. Selective superior mesenteric arteriography demonstrated both an early-draining vein and several small collections of contrast material within the region of the cecum; these were thought to be vascular ectasias (Fig 1).

The patient underwent right-sided hemicolectomy for removal of the vascular ectasias. As described by Mitsudo and associates, ${ }^{1}$ the major arteries and veins of the pathologic specimen were injected with silicone rubber compound to aid in identification. Examination of the 


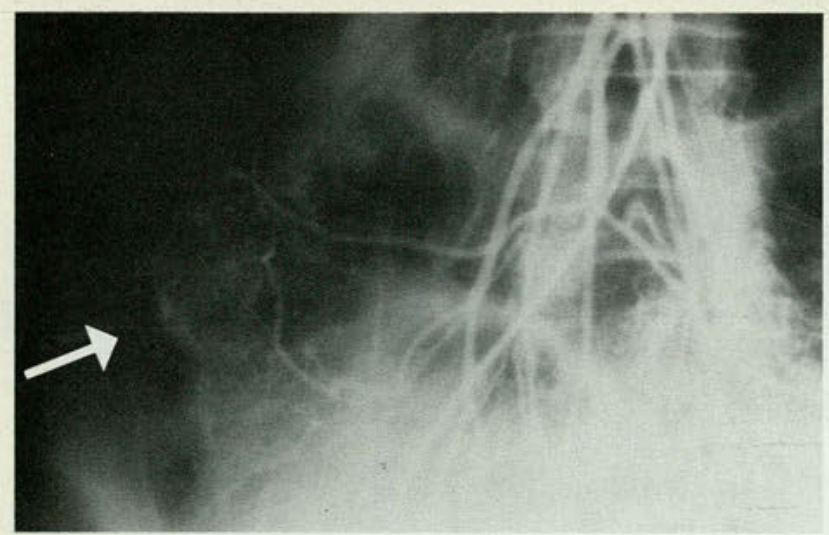

Fig 1. Selective superior mesenteric arteriogram shows earlyfilling vein (arrow).

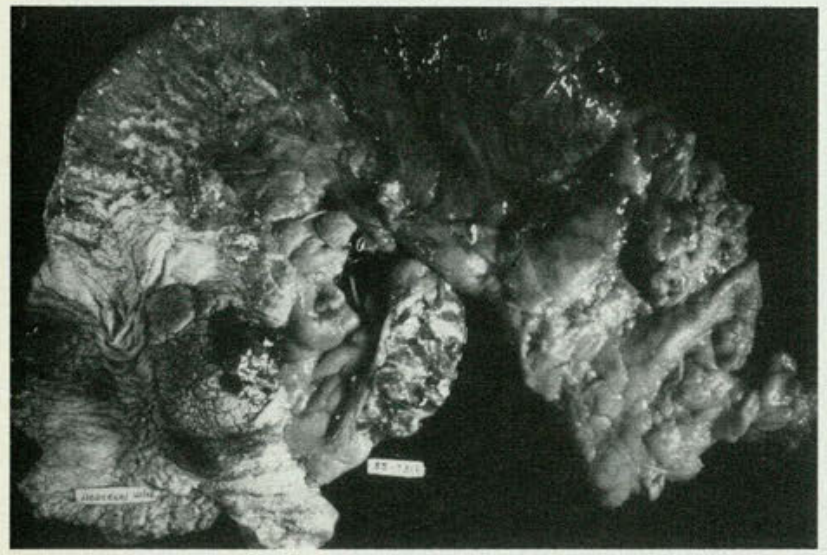

Fig 2. Silicone-compound-injected hemicolectomy specimen. Vascular ectasias are clearly visible and adjacent to ileocecal valve.

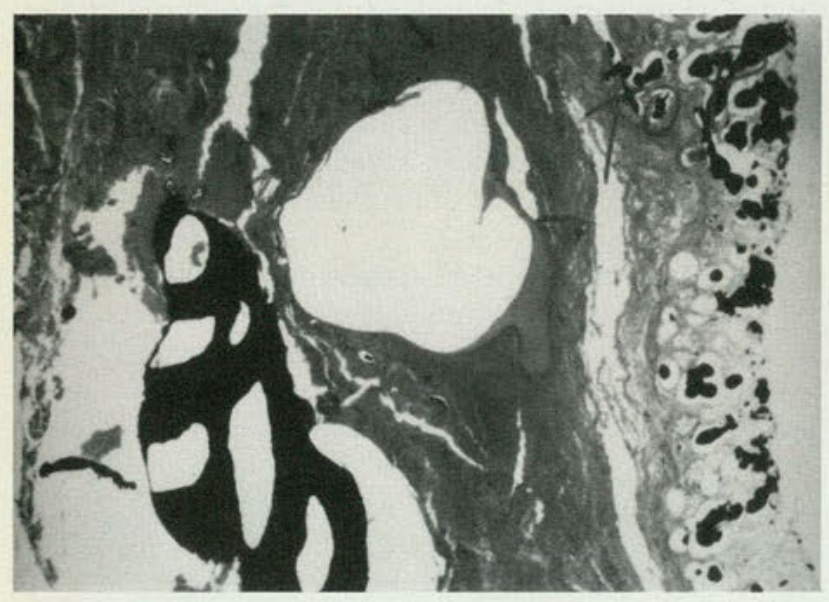

Fig 3. Vascular ectasia composed of both dilated submucosal veins (one containing silicone compound) and ectatic mucosal vessels (hematoxylin and eosin stain, original magnification $\times 40$ )

gross specimen revealed a cluster of several vascular lesions measuring $6 \mathrm{~cm}$ aggregately just distal to the ileocecal valve (Fig 2). Dilated submucosal blood vessels also were observed near the colonic margin of resection.

Microscopic examination demonstrated dilated, tor- tuous submucosal and mucosal blood vessels, and a diagnosis of multiple vascular ectasias was made (Fig 3 ). The patient had a satisfactory postoperative course and has had no recurrence of intestinal bleeding during a follow-up period of 18 months.

\section{Discussion}

There has been a slowly evolving and changing perception of the causes of lower intestinal bleeding over the past 60 years. Cancer of the large bowel was thought to be the most common cause in the 1920 s, while in the 1940 s and 1950 s, diverticulitis and diverticulosis were recognized increasingly as causes of often brisk, frequently life-threatening intestinal hemorrhage. ${ }^{3}$ Diverticulosis, particularly when it involves the right side of the colon, ${ }^{2}$ still is thought to be a major cause of lower intestinal bleeding, and some authors think is the most common cause.

In a review by Boley and associates, ${ }^{3}$ diverticulosis was by far the most frequent discharge diagnosis for patients who had major lower intestinal hemorrhage on admission (Montefiore Hospital and Medical Center, New York, 1971 to 1976). However, localization of the bleeding site to a colonic diverticulum is difficult, and the diagnosis of hemorrhage secondary to diverticular disease is made largely by exclusion.

Quinn formalized this concept many years ago by denoting five criteria for attributing lower intestinal bleeding to diverticular disease, as follows: passage of considerable maroon or bright red stools; roentgenographic evidence of diverticuli; barium enema or sigmoidoscopic elimination of other causes of hemorrhage; negative upper gastrointestinal series or negative gastric aspirate, or both; and normal coagulation studies. ${ }^{3}$ Interestingly, in the review by Boley and associates, none of the patients who were diagnosed as having hemorrhage from diverticular disease met all five of Quinn's criteria; less than half met four criteria, and one fourth had another possible source of bleeding.

\section{Vascular ectasias}

Vascular ectasias of the colon on the right side in elderly patients first were detected and recognized as a possible cause of lower intestinal bleeding in 1960 by Margulis and coworkers. ${ }^{4}$ They visualized the vascular lesion using intraoperative angiography. Since that time, selective mesenteric angiography, radionuclide blood loss scanning, ${ }^{5}$ and fiberoptic colonoscopy, ${ }^{6,7}$ have been used with increasing success to detect vascular ectasias. Each of these techniques allows visualization of the lesion and can localize the source of active bleeding prior to laparotomy. 
The current mainstay of therapy is right hemicolectomy, which has been curative in $80 \%$ to $90 \%$ of cases when hemorrhage had been localized to the colon on the right preoperatively. ${ }^{3}$

Incidence. There are indications that vascular ectasias are quite common in the elderly population. In a small study ${ }^{8}$ of elderly patients who underwent right-sided hemicolectomy for carcinoma and no clinical episodes of bleeding, vascular ectasias were detected in $53 \%$ of the resected, siliconeinjected specimens. Researchers using autopsy series have had limited success in examining injected specimens because of postmortem mucosal autolysis and loss of vascular integrity..$^{9}$ Only larger and more advanced vascular ectasias are detected angiographically; therefore, rates of occurrence in radiographic series underestimate the true incidence of the lesions.

Pathologic findings. One of the difficulties in studying right-sided colonic vascular ectasias has been identifying the lesions in surgically resected specimens. One approach has been to inject the major mesenteric arteries and veins with a silicone rubber compound using plastic intravenous catheters. ${ }^{1}$ The silicone compound is allowed to polymerize and solidify overnight. The specimen is then opened and fixed in formalin. After fixation, histologic sections can be prepared in the usual manner.

We used this procedure for handling the hemicolectomy specimen from our patient, and the injection technique made the vascular lesion readily apparent against the background of the surrounding colonic mucosa. The procedure is facilitated by having the mesenteric vessels tagged by the surgeon during operation.

Vascular ectasias, which may occur singly or in multiples, vary in dimension from $0.1 \mathrm{~cm}$ to $1 \mathrm{~cm}$. Boley and colleagues ${ }^{8}$ found as many as 50 individual vascular ectasias within a single surgical specimen.

The lesions are located in the cecum and ascending colon in the majority of cases, but they also may be found in the transverse colon or, rarely, in the descending colon. Weaver and coauthors ${ }^{9}$ have described vascular lesions of the gastric antrum and duodenum that endoscopically and histologically resemble those more commonly seen in the right-sided colon.

When visualized in a silicone-injected surgical specimen, vascular ectasias are a slightly raised tuft of tortuous mucosal blood vessels with a "coral reef' appearance. There may be adjacent mucosal or submucosal hemorrhage, which can aid in iden- tification. Rarely, vascular ectasias have been found within the walls of right-sided colonic diverticuli. $^{8}$

Histologically, the primary finding is the presence of dilated, tortuous, submucosal veins. The veins are thin walled and lack the thickened, 'arterialized' wall that often is seen in arteriovenous malformations at other anatomic sites. The intestinal vascular lesions in hereditary hemorrhagic telangiectasia (Rendu-Osler-Weber syndrome) also contain dilated tortuous veins, but these veins surround a central artery and are intramucosal. ${ }^{10,11}$

The more advanced vascular ectasias in elderly persons also may contain dilated mucosal venules and capillaries, but not without the presence of dilated submucosal veins. In some cases, microscopic sections of these lesions demonstrate only a single cell layer of colonic epithelium and capillary basement membrane separating the dilated mucosal vessels from the colonic lumen. It is apparent that even minor mucosal trauma could initiate intestinal bleeding.

Pathogenesis. Boley and colleagues ${ }^{8}$ presented convincing evidence that vascular ectasias in elderly persons are degenerative lesions rather than congenital malformations. They hypothesize that longterm, low-grade, partial, intermittent obstruction of draining veins at the level of the muscle wall of the bowel eventually leads to nonreversible dilatation and tortuosity of the submucosal veins. As the obstruction continues, venules and capillaries in the mucosa also expand. Eventually, the increased venous and capillary pressures may overcome the resistance of precapillary sphincters and create microscopic arteriovenous malformations. This proposed progression correlates well with angiographic findings. The early-filling vein seen on the angiogram probably represents late vascular ectasia, in which precapillary sphincters are incompetent and, thus, allow this early filling of mucosal veins.

The preponderance of vascular ectasias in the cecum and ascending colon may be related to a basic principle of physics. Laplace's law states that wall tension in a rigid tube is directly proportional to the diameter and the intraluminal pressure. ${ }^{1}$ In the colon, the cecum has the greatest diameter by far, and, therefore, it may have the greatest wall tension. The portion of bowel with the greatest wall tension would be expected to cause the greatest intramural venous obstruction.

Association with aortic stenosis. It has been observed $^{12-18}$ repeatedly that there is an association between lower intestinal bleeding and aortic 
stenosis in the elderly population. The colonic lesions responsible for hemorrhage often were not identified. When the bleeding could be localized, the cecum and ascending colon were the most common sites. Since the characterization of colonic vascular ectasias, it is clearer that the true association is between aortic stenosis and vascular ectasias. Boley and colleagues ${ }^{8}$ found that $20 \%-25 \%$ of patients with colonic vascular ectasias also had aortic stenosis. Cody and associates ${ }^{17}$ convincingly demonstrated that the association between these two entities was much greater than would be expected by chance alone.

It is unclear whether aortic stenosis and the resulting hemodynamic changes have any bearing on the formation of vascular ectasias. Boley and associates $^{8}$ contend that the lower perfusion and pulse pressure in patients with aortic stenosis may increase the risk of bleeding from vascular ectasias because of ischemic necrosis of the colonic epithelium between ectatic mucosal vessels and the colonic lumen. They do not think that the circulatory effects of aortic stenosis have any bearing on the formation of vascular ectasias.

Williams ${ }^{16}$ raised the possibility that a congenitally deformed aortic valve may be associated with vascular ectasias, and he assumed that the colonic vascular lesion also was a congenital anomaly. In Cody and coauthors'17 series of patients who had undergone cardiac surgery for aortic valve replacement, the resected valves demonstrated calcification of the senile type; no cases of congenital bicuspid or unicuspid aortic valves were found. This evidence supports Boley and colleagues' ${ }^{8}$ contention that colonic vascular ectasias are an acquired, degenerative lesion of aging.

\section{Association with von Willebrand's disease.} Cases of concurrent colonic vascular ectasia and acquired von Willebrand's disease also have been reported. Acquired von Willebrand's disease is rare. In each of two reported cases, ${ }^{19,20}$ the patient had monoclonal gammopathy and no detectable circulating factor VIII inhibitors. One patient had a colonic vascular lesion that appeared to be consistent with hereditary hemorrhagic telangiectasia endoscopically, and the other had a gastric vascular ectasia. Therefore, to date, the association of acquired von Willebrand's disease with typical right-sided colonic vascular ectasias in elderly persons is very tenuous.

An interesting speculation can be made about the coexistence of these diseases, however. Because the factor VIII complex is synthesized partially by vascular endothelium, perhaps an underlying endothelial defect is responsible for the pathogenesis of both lesions.

\section{Summary}

The case presentation illustrates several important points in the diagnosis and management of intestinal hemorrhage in elderly patients, as follows: (1) The most obvious colonic lesion in a bleeding patient may not be the only source of hemorrhage; (2) angiography and colonoscopy play important roles in localizing intestinal bleeding of obscure cause; and (3) there is an association between colonic vascular ectasias and aortic stenosis.

Increased awareness of colonic vascular ectasias should lead to earlier diagnosis and improved clinicopathologic correlation.

1. Mitsudo SM, Boley SJ, Brandt LJ, et al: Vascular ectasias of the right colon in the elderly: A distinct pathologic entity. Hum Pathol 1979;10:585600 .

2. Casarella WJ, Kanter IE, Seaman WB: Right-sided colonic diverticula as a cause of acute rectal hemorrhage. N Engl J Med 1972;286:450-453. 3. Boley SJ, DiBiase A, Brandt LJ, et al: Lower intestinal bleeding in the elderly. Am J Surg 1979;137:57-64.

4. Margulis AR, Heinbecker P, Bernard HR: Operative mesenteric arteriography in the search for the site in bleeding in unexplained gastrointestinal bleeding. Surgery 1960;48:534-539.

5. Harris RA: Radionuclide evaluation of lower gastrointestinal hemorrhage: A review. JAOA 1986;86:226-234.

6. Skibba RM, Hartong WA, Mantz FA, et al: Angiodysplasia of the cecum: Colonoscopic diagnosis. Gastrointest Endosc 1976;22:177-179.

7. Rogers BHG, Adler F: Hemangiomas of the cecum: Colonoscopic diagnosis and therapy. Gastroenterology 1976;71:1079-1083.

8. Boley SJ, Sammartano RJ, Adams A: On the nature and etiology of vascular ectasias of the colon: Degenerative lesions of aging. Gastroenterology 1977;72:650-660.

9. Weaver GA, Alpern HD, Davis JS, et al: Gastrointestinal angiodysplasia associated with aortic valve disease: Part of a spectrum of angiodysplasia of the gut. Gastroenterology 1979;77:1-11.

10. Baer JW, Ryan S: Analysis of cecal vasculature in the search for vascular malformations. AJR 1976;126:394-405.

11. Bird RM, Jaques WE: Vascular lesions of hereditary hemorrhagic telangiectasia. N Engl J Med 1959;260:597-599.

12. Heyde EC: Gastrointestinal bleeding in aortic stenosis, letter. NEngl $J$ Med 1958;259:196.

13. Schwartz BM: Additional note on bleeding in aortic stenosis, letter. N Engl J Med 1958;259:456.

14. Castleman B, McNeely BU: Case record 49-1965. N Engl J Med 1965;273:1096-1105.

15. Galloway SJ, Casarella WJ, Shimkin PM: Vascular malformation of the right colon as a cause of bleeding in patients with aortic stenosis. Radiology 1974;113:11-15.

16. Williams RC Jr.: Aortic stenosis and unexplained gastrointestinal bleeding. Arch Intern Med 1961;108:859-863.

17. Cody MC, O'Donovan TP, Hughes RW Jr.: Idiopathic gastrointestinal bleeding and aortic stenosis. Am J Dig Dis 1974;19:393-398.

18. Boss EG Jr, Rosenbaum JM: Bleeding from the right colon associated with aortic stenosis. Am J Dig Dis 1971;16:269-275.

19. Rosborough TK, Swaim WR: Acquired von Willebrand's disease, platelet-release defect and angiodysplasia. Am J Med 1978;65:96-100.

20. Cass AJ, Bliss BP, Bolton PP, et al: Gastrointestinal bleeding, angiodysplasia of the colon and acquired von Willebrand's disease. $\mathrm{Br}$ J Surg 1980;67:639-641.

From the Department of Pathology and Laboratory Medicine, Doctors Hospital, Columbus, Ohio.

Reprint requests to Dr Jenkinson, Doctors Hospital, 1087 Dennison Ave, Columbus, OH 43201. 